\title{
Contraintes résiduelles de relaxation dans la croissance hétéroépitaxiale des films minces
}

\section{Residual stresses relaxation in heteroepitaxial growth of thin film}

\author{
Aziz Soufi ${ }^{1}$, Khalil El-Hami ${ }^{2}$ \\ ${ }^{1}$ University of Hassan $1^{\text {st }}$, National school of Applied Sciences of Khouribga, Laboratory of Nanosciences and Modeling, \\ Khouribga, Morocco.az_sfi@yahoo.fr \\ ${ }^{2}$ University of Hassan $1^{\text {st }}$, Faculty of Khouribga,Laboratory of Nanosciences and Modeling, Khouribga, Morocco. \\ khalil.elhami@uhp.ac.ma
}

RÉSUMÉ. La dynamique de la nucléation des dislocations des dipôles de bord à partir des zones latérales libres, à proximité des interfaces entre le substrat et la couche mince du film obtenues par croissance hétéroépitaxiale est discutée. La méthode d'analyse utilise la superposition des dislocations d'image et de la distribution des forces de surface de Boussinesq. Le calcul théorique est effectué en utilisant la méthode du gradient conjugué et le code Mathematica. Ce travail montre comment la stabilité des dislocations de bord, disloquées à partir de la surface latérale, est très importante pour évacuer les déformations entre les paramètres des mailles.

ABSTRACT. The dynamic of edge dipoles dislocations nucleation's from free lateral areas, near the thin film-substrate interfaces obtained by heteroepitaxial growth is discussed. The analysis method uses the superposition of image dislocations and Boussinesq surface forces distribution. The theoretical calculation is carried out using the conjugate gradient method and Mathematica code. This work reveals how the stability of edge dislocations dipoles, nucleated from lateral surface is very important to evacuate strains Misfit between mesh parameters.

MOTS-CLÉS. hétéroépitaxie, film mince, dislocation de l'interface, forces de l'image, forces de Boussinesq, dipôle de dislocation.

KEYWORDS. heteroepitaxy, thin film, interface dislocation, image forces, Boussinesq forces, dislocation dipole.

\section{Introduction}

The introduction of edge dislocation from free areas as precipitates, islands, steps etc... plays a capital role to relax stresses Misfit discord between thin film and substrate [1].

It's also demonstrated that the thickness $[2,3]$ of the buried thin film embedded in the material matrix and the hetero-epitaxial deformation $\frac{\delta a}{a}=\frac{a_{\text {subs }}-a_{\text {thin }}}{a_{\text {subs }}}$ play a major role to stabilize interfaces. But the introduction of a single dislocation or a stack of $\mathrm{n}$ dislocations of the same nature (Burgers vectors with the same signs) does not completely solve the problem. The limit of similar a solution is caused by the instability of the dislocations which conditioned by a critical thickness he which must not be exceeded. In the contrary case, the dislocations risk leaving the materials at the interface one after the other causing its interruption, similar a situation will cause the degradation of its physical properties (electrical, magnetic, optical, or other ...).

To adjust this problem of emergence, we propose in this work to introduce a stack edge dislocations dipoles $( \pm b)$ [4] from a free lateral surface [5].

\section{The Problem of Boussinesq}

The Boussinesq problem consists in studying a semi-infinite unit subjected to a ponctuel and a normal load on the plane which limits it [6], it's largely and regular used in the situations of contact. The Boussinesq forces are weak enough to apply the theory of linear elasticity. The contact on the 
lateral surface is considered to be small in comparison with the radii of curvature for the undeformed surface. The contact stresses are strongly concentrated near the contact zone and decrease rapidly in intensity at a distance from the point of contact, the practical region of interest is located close to the contact interface. The strains are calculated with a good approximation considering each body as a semi-infinite elastic solid delimited by a flat lateral surface. We consider our study material (substratefim) as a matrix of an elastic half-space charged in a one-dimensional mode and on a very restricted band. In our study, the lateral boundary surface at $x=0$ is the plane $y-z$ and the axis Ox is oriented normally to the surface towards the outside of the material. The charged surface is parallel to the solid matrix of the material, infinite in the lateral direction $\mathrm{Oz}$ and has its useful thickness limited in the direction $\mathrm{Oy}$, which are function only of $\mathrm{x}$ and $\mathrm{y}$.

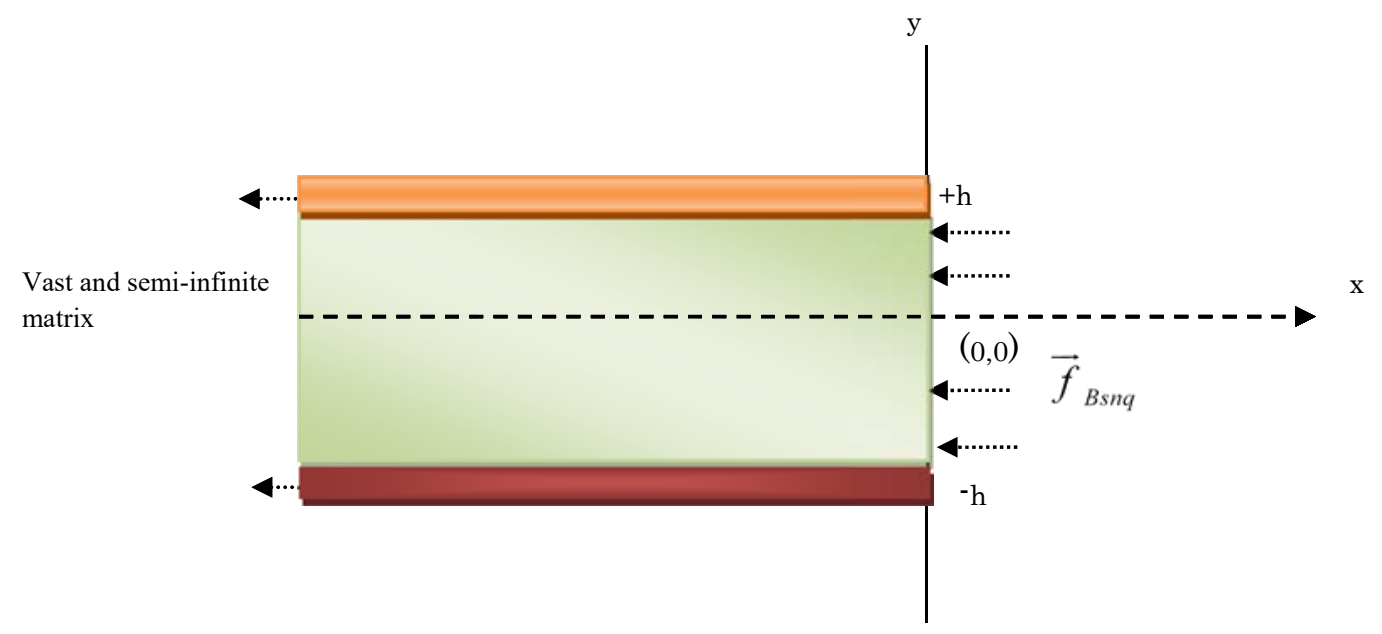

Figure 1. The structure of the material studied, with the application of Boussinesq forces

\section{Distribution of Boussinesq forces}

Boussinesq forces with unit modulus $\mathrm{Q}=1$ are applied at a point on a plane and free surface. We shall distinguish the normal Boussinesq forces and the tangential Boussinesq forces.

For point forces originally exerted on a free surface perpendicular to the ox axis. The stresses induced in the solid can be obtained from the corresponding Airy functions $\phi_{i}$, deduced from the theory of the potentials of Boussinesq:

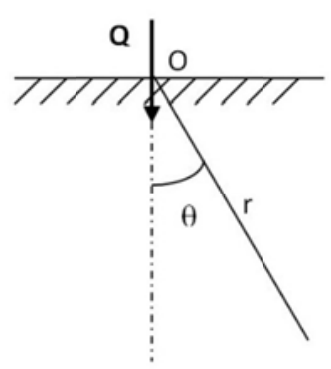

Figure 2. The normal force's Boussinesq

Normal Forces:

$$
\left\{\begin{array}{l}
\phi_{n}(x, y)=\frac{-y}{\pi} \cdot \arctan \frac{y}{x}=\frac{-1}{\pi} \cdot \theta \cdot r \cdot \sin \theta \\
\phi_{n}(r, \theta)=A \cdot \theta \cdot r \cdot \sin \theta \\
A=C^{t e}=\frac{-1}{\pi}
\end{array}\right.
$$


Tangential forces

$$
\left\{\begin{array}{l}
\phi_{t}(x, y)=\frac{x}{\pi} \cdot \arctan \frac{y}{x}=\frac{1}{\pi} . . r \cdot \theta \cdot \cos \theta \\
\phi_{t}(r, \theta)=-A \cdot . r \cdot \theta \cdot \cos \theta
\end{array}\right.
$$

Normal and tangential stresses: cylindrical coordinates

$$
\text { [7] }\left\{\begin{array}{l}
\sigma_{r r}^{n}=\frac{1}{r} \cdot \frac{\partial \phi_{t}(r, \theta)}{\partial r}+\frac{1}{r^{2}} \cdot \frac{\partial^{2} \phi_{t}(r, \theta)}{\partial \theta^{2}}=\frac{2 \cdot A \cdot \cos \theta}{r} \\
\sigma_{\theta \theta}^{n}=\frac{\partial^{2} \phi_{t}(r, \theta)}{\partial r^{2}}=0 \\
\tau_{r \theta}^{n}=\frac{-\partial}{\partial r}\left(\frac{1}{r} \cdot \frac{\partial \phi_{t}(r, \theta)}{\partial \theta}\right)=0
\end{array}\right.
$$$$
\left\{\begin{array}{l}
\sigma_{r r}^{t}=\frac{1}{r} \cdot \frac{\partial \phi_{t}(r, \theta)}{\partial r}+\frac{1}{r^{2}} \cdot \frac{\partial^{2} \phi_{t}(r, \theta)}{\partial \theta^{2}}=\frac{2 \cdot A \cdot \sin \theta}{r} \\
\sigma_{\theta \theta}^{t}=\frac{\partial^{2} \phi_{t}(r, \theta)}{\partial r^{2}}=0 \\
\tau_{r \theta}^{t}=\frac{-\partial}{\partial r}\left(\frac{1}{r} \cdot \frac{\partial \phi_{t}(r, \theta)}{\partial \theta}\right)=0
\end{array}\right.
$$
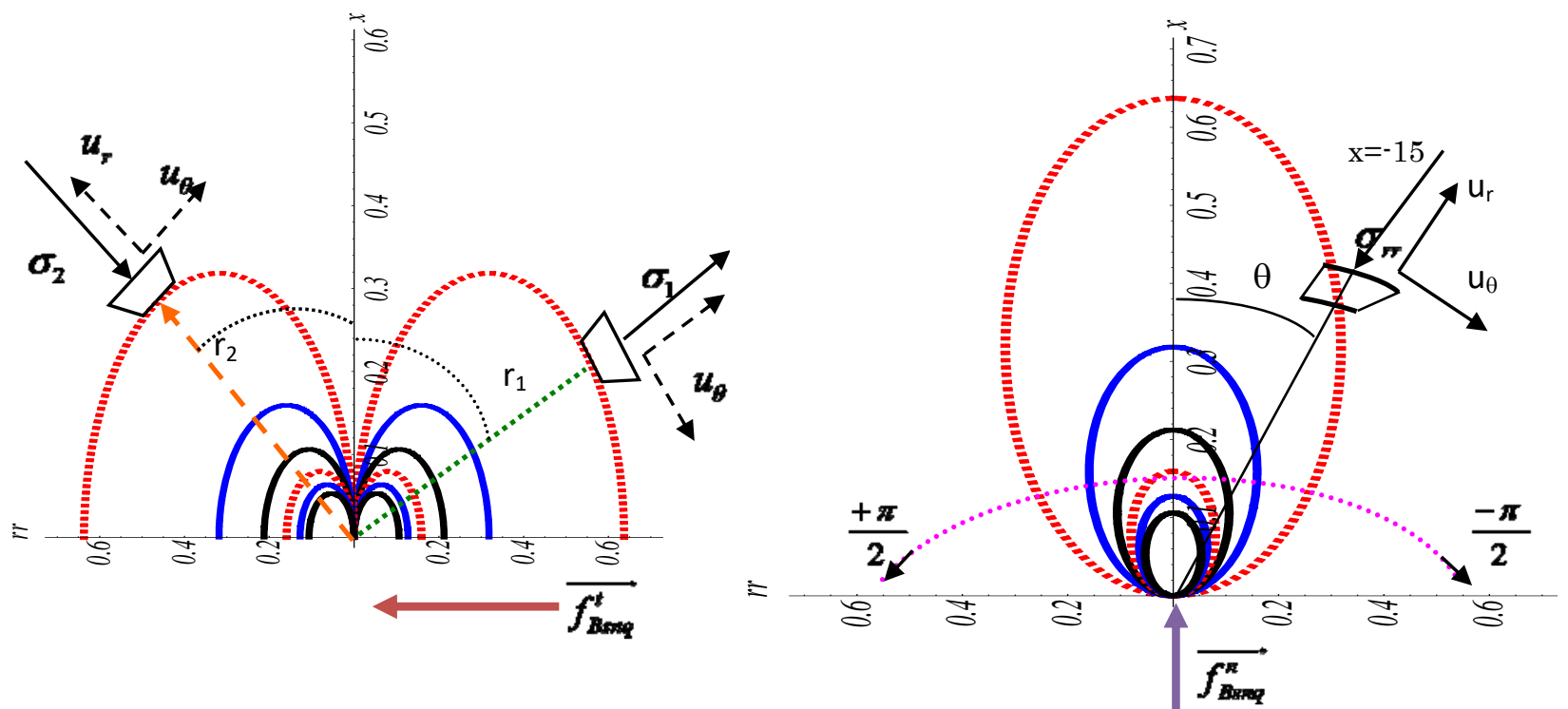

Figure 3. $\operatorname{orr}(\operatorname{tg})$ function of the orthoradial variable $\theta$ Figure 4. $\mathrm{\sigma rr}($ norm) function of the orthoradial variable $\theta$

System of stresses reflecting the radial distribution detected around the point $\mathrm{O}$ of the concentrated unit force with very important key, at a great distance from the point of application $(r \rightarrow \infty)$ the stress field tends to zero: $\theta= \pm \frac{\pi}{2}\left\{\begin{array}{l}\sigma_{\theta}^{n}=\tau_{r \theta}^{n}=0 \\ \sigma_{r r}^{n}=0\end{array}\right.$.

And for the tangential stress field: $\theta= \pm \frac{\pi}{2}\left\{\begin{array}{l}\sigma_{\theta}^{t}=\tau_{r \theta}^{t}=0 \\ \sigma_{r r}^{t} \neq 0\end{array}\right.$

The radial stress at the surface becomes tangential, which makes the tangential Boussinesq forces very important to cancel the tangential stress fields (direct and images) on the free lateral surface of the material under study. 


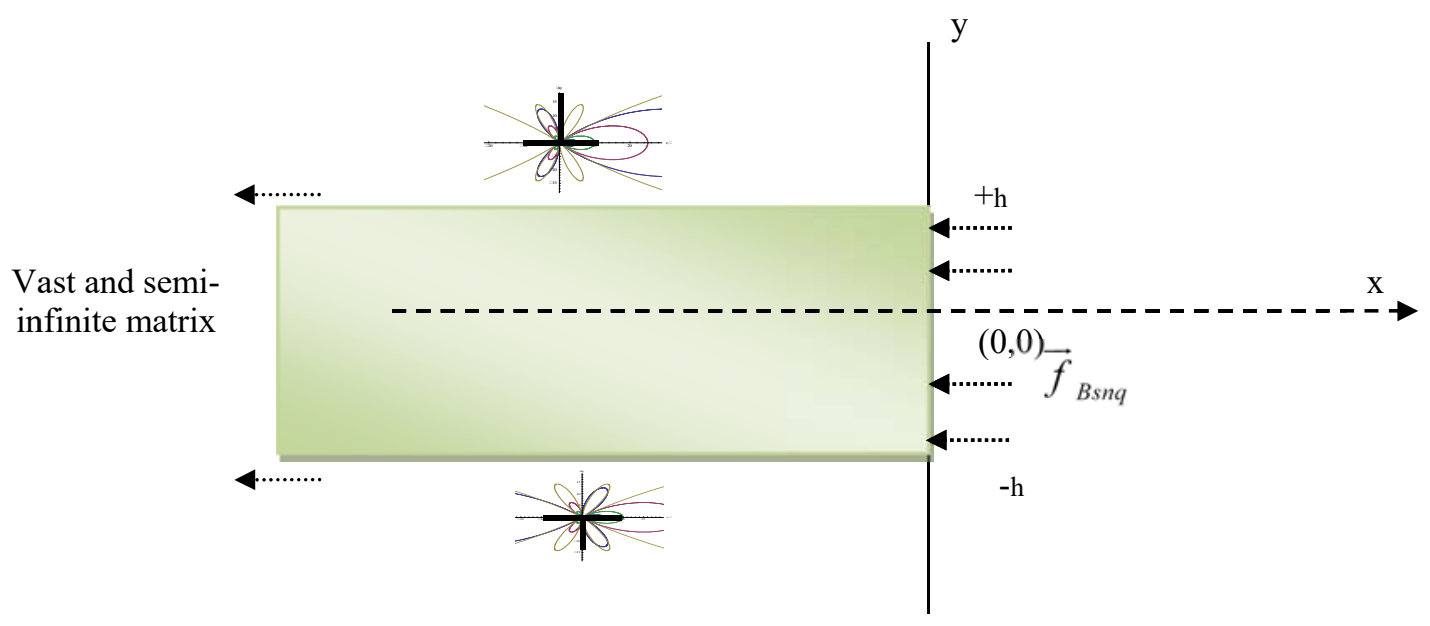

Figure 5. Dipole nucleation of edge dislocations

Surroundings the origin $x=0^{ \pm}$the stresses are coming the following values:

$$
\left\{\begin{aligned}
\sigma_{x x}^{n} & \rightarrow \frac{-x}{|x|} \cdot \delta(y) \\
\sigma_{y y}^{n} & \rightarrow \frac{-x}{|x|} \cdot \delta(y) \\
\tau_{x y}^{n} & \rightarrow 0
\end{aligned}\right.
$$$$
\left\{\begin{aligned}
\sigma_{x x}^{t} & \rightarrow 0 \\
\sigma_{y y}^{t} & \rightarrow \frac{-1}{\pi \cdot y} \\
\tau_{x y}^{t} & \rightarrow \frac{-x}{|x|} \cdot \delta(y)
\end{aligned}\right.
$$

These singularities will be placed on the outer free surface of the solid. We place them at the points $\left(\mathrm{x}^{\prime}=\varepsilon, \mathrm{y}^{\prime}\right)$ and make $\varepsilon$ towards $0^{+}$.

The stresses applied by singularities are obtained by replacing in equations (7) and (8) x by $x-x$ 'and y by $y-y^{\prime}$.

$$
\left\{\begin{array} { l } 
{ \sigma _ { x x } ^ { n } = \frac { \partial ^ { 2 } \phi _ { n } ( x , y ) } { \partial y ^ { 2 } } = \frac { - 2 \cdot x ^ { 3 } } { \pi \cdot ( x ^ { 2 } + y ^ { 2 } ) ^ { 2 } } } \\
{ \sigma _ { y y } ^ { n } = \frac { \partial ^ { 2 } \phi _ { n } ( x , y ) } { \partial x ^ { 2 } } = \frac { - 2 \cdot x \cdot y ^ { 2 } } { \pi \cdot ( x ^ { 2 } + y ^ { 2 } ) ^ { 2 } } } \\
{ \tau _ { x y } ^ { n } = \frac { - \partial ^ { 2 } \phi _ { n } ( x , y ) } { \partial x \partial y } = \frac { - 2 \cdot x ^ { 2 } \cdot y } { \pi \cdot ( x ^ { 2 } + y ^ { 2 } ) ^ { 2 } } ( 7 ) }
\end{array} \left\{\begin{array}{l}
\sigma_{x x}^{t}=\frac{\partial^{2} \phi_{t}(x, y)}{\partial y^{2}}=\frac{-2 \cdot x^{2} \cdot y}{\pi \cdot\left(x^{2}+y^{2}\right)^{2}} \\
\sigma_{y y}^{t}=\frac{\partial^{2} \phi_{t}(x, y)}{\partial x^{2}}=\frac{-2 \cdot y^{3}}{\pi \cdot\left(x^{2}+y^{2}\right)^{2}} \\
\tau_{x y}^{t}=\frac{-\partial^{2} \phi_{t}(x, y)}{\partial x \partial y}=\frac{-2 \cdot x \cdot y^{2}}{\pi \cdot\left(x^{2}+y^{2}\right)^{2}}(8)
\end{array}\right.\right.
$$

So we have: $\left\{\begin{array}{l}\lim _{\varepsilon \rightarrow 0^{+}} \sigma_{i j}\left(-\varepsilon, y-y^{\prime}\right) \\ \lim _{x \rightarrow 0^{-}} \sigma_{i j}\left(x, y-y^{\prime}\right)\end{array}\right.$

For the singularities located on the outer surface at $\mathrm{y}$ ', the normal and tangential forces are:

$$
\left\{\begin{array}{l}
\sigma_{x x}^{n}(0, y) \rightarrow \delta\left(y-y^{\prime}\right) \\
\tau_{x y}^{n}(0, y) \rightarrow 0 \\
\sigma_{x x}^{t}(0, y) \rightarrow 0 \\
\tau_{x y}^{t}(0, y) \rightarrow \delta\left(y-y^{\prime}\right)
\end{array}\right.
$$


Then, to cancel the forces on the surface $\mathrm{x}=0$, a Boussinesq tangential distribution forces is added to the image dislocation.

The stresses of this Boussinesq distribution are represented by: $\sum_{i j}^{\xi}(x, y)$

So, the total stresses at a point $(\mathrm{x}, \mathrm{y})$ are: $\sigma_{i j}^{\text {tot }}(x, y)=\sigma_{i j}^{\text {direct }}(x, y)+\sum_{i j}^{\xi}(x, y)+\sigma_{i j}^{i m a g e}(x, y){ }_{(9)}$

With: $\sigma_{i j}^{\text {direct }}(x, y)$ Field of direct stresses' dislocations

And $\sigma_{i j}^{\text {image }}(x, y)$ Field of stresses' images dislocations

Forces applied on the free surface $\mathrm{x}=0: \overrightarrow{d F}=(\Sigma) \overrightarrow{d S}=\left[\begin{array}{ccc}\sigma_{x x} & \sigma_{x y} & 0 \\ \sigma_{x y} & \sigma_{y y} & 0 \\ 0 & 0 & \sigma_{z z}\end{array}\right]\left(\begin{array}{c}d y d z \\ 0 \\ 0\end{array}\right)=\left(\begin{array}{c}\sigma_{x x} d y d z \\ \sigma_{x y} d y d z \\ 0\end{array}\right)$

The field of edge dislocation stresses at the origin with Burgers vector $(b, 0)$ in an infinite space [8] are given by: $\left\{\begin{array}{l}\sigma_{x x}{ }^{f}=\frac{-D \cdot y \cdot\left(3 x^{2}+y^{2}\right)}{\left(x^{2}+y^{2}\right)^{2}} \\ \sigma_{y y}{ }^{f}=\frac{D \cdot y \cdot\left(x^{2}-y^{2}\right)}{\left(x^{2}+y^{2}\right)^{2}} \\ \sigma_{x y}{ }^{f}=\frac{D \cdot x \cdot\left(x^{2}-y^{2}\right)}{\left(x^{2}+y^{2}\right)^{2}} \\ D=\frac{\mu \cdot b}{2 \cdot \pi \cdot(1-v)}\end{array}\right.$

Under the single effect of the image dislocation the normal force vanishes: $\sigma_{x x} d y d z \overrightarrow{e_{x}}=\overrightarrow{0}$ (12)

While the shear force doubles its value: $2 \sigma_{x y} d y d z \overrightarrow{e_{y}}=2 \cdot \frac{\mu \cdot b \cdot a}{2 \cdot \pi \cdot(1-v)} \cdot \frac{a^{2}-y^{2}}{\left(a^{2}+y^{2}\right)^{2}} \overrightarrow{e_{y}}$

To cancel the total force at $\mathrm{x}=0$, an image dislocation and a tangential Boussinesq force distribution are added to the direct dislocation (d).

At a point $(\mathrm{x}, \mathrm{y}):\left\{\begin{array}{l}\sum_{i j}{ }^{\xi}(x, y)=\int \xi\left(y^{\prime}\right) \cdot \sigma_{i j}{ }^{t}\left(x-\varepsilon, y-y^{\prime}\right) d y^{\prime} \\ \sigma_{i j}{ }^{\text {tot }}(x, y)=\int \xi\left(y^{\prime}\right) \cdot \sigma_{i j}{ }^{t}\left(x-\varepsilon, y-y^{\prime}\right) d y^{\prime}+\sigma_{i j}{ }^{d}(x+a, y)+\sigma_{i j}{ }^{i m a g e}(x-a, y) \\ \varepsilon \rightarrow 0^{+}\end{array}\right.$

$$
\sigma_{i j}{ }^{\text {tot }}(0, y)=\int \xi\left(y^{\prime}\right) \cdot \delta\left(y-y^{\prime}\right) d y^{\prime}+2 \cdot D \cdot a \cdot \frac{a^{2}-y^{2}}{\left(a^{2}+y^{2}\right)}=0
$$

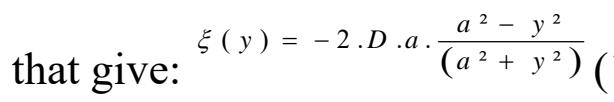

Using the Fourier transform we compute the Airy function of the corresponding Boussinesq forces: $\left\{\begin{array}{l}\phi^{B s n q}(x, y)=\int \xi\left(y^{\prime}\right) \cdot \phi_{t}\left(x, y-y^{\prime}\right) d y^{\prime} \\ \phi^{B s n q}(x, k)=\xi(k) \cdot \phi_{t}(x, k)=-2 . i \cdot D \cdot \pi \cdot a \cdot x \cdot \frac{|k|}{k} \cdot \exp (|k| \cdot(a-x))\end{array}\right.$ 
$\phi^{B s n q}(x, y)=\frac{2 \cdot D \cdot a \cdot x \cdot y}{(x-a)^{2}+y^{2}}$

$\tau_{x y}^{B s n q}(x, y)=\frac{-\partial^{2} \phi^{B s n q}(x, y)}{\partial x \partial y}=\frac{2 \cdot D \cdot(-a)}{\left[(a-x)^{4}+2 \cdot x \cdot(a-x)^{3}-6 \cdot x \cdot(a-x) \cdot y^{2}-y^{4}\right]}$

The graph of $\tau_{x y}^{\text {Bsnq }}(x, y)$ for different values of $\mathrm{x}$.

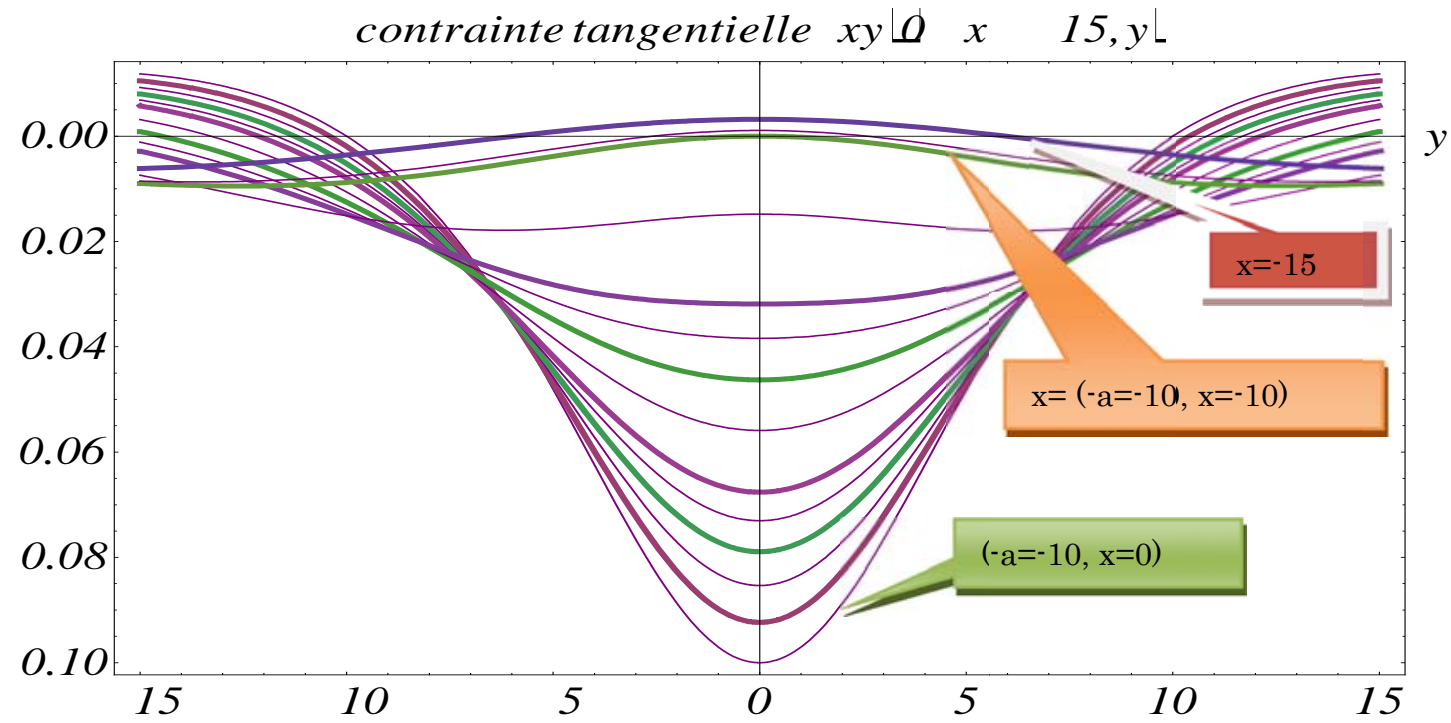

Figure 7. Graph of the Boussinesq shear stress

In locality of $\mathrm{y}=0$ and with condition $|x| \leq a$, the Boussinesq distribution cancels the tangential stress: $\tau_{x y}^{\text {tot }}(0, y)=\tau_{x y}^{\text {direct }}(0, y)+\tau_{x y}^{\text {image }}(0, y)=\frac{2 \cdot D \cdot\left(a^{2}-y^{2}\right)}{\left(a^{2}+y^{2}\right)^{2}}(18)$

But this cancellation has a limit: for $\mathrm{x}=-15$, the cancellation is no longer valid and the surface is no longer free.

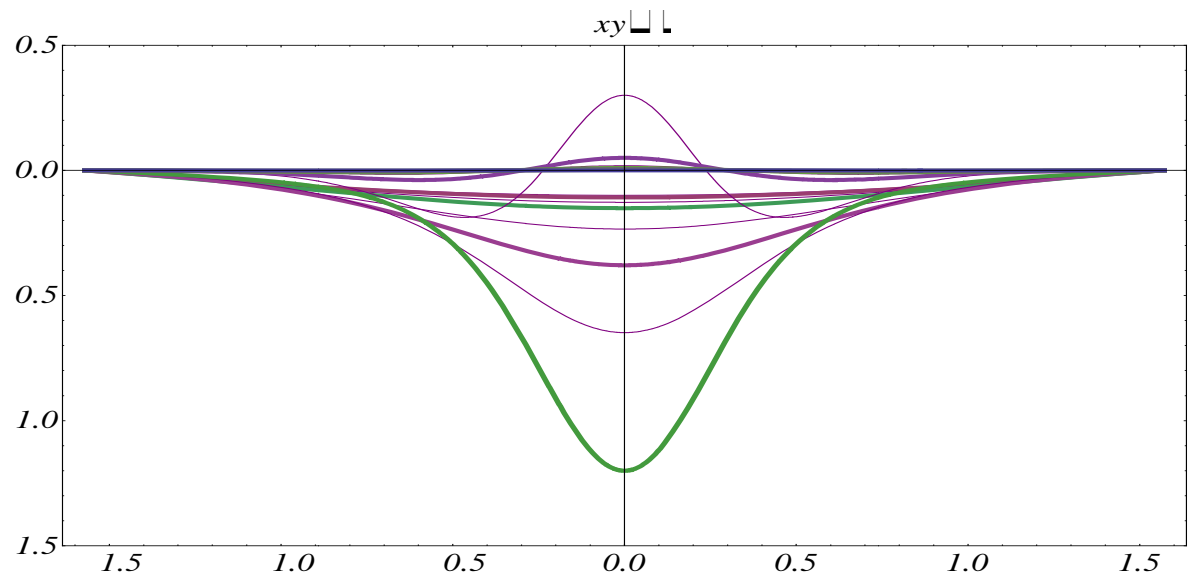

Figure 8. Illustration following the variable $\theta: \tau_{x y}^{B s n q}=f\left(\frac{-\pi}{2} \leq \theta \leq \frac{\pi}{2}\right) ; \theta=\arctan \frac{y}{x}$ 
As a result:

When, $\mathrm{x}$ and $\mathrm{y}$ are such that $|\theta| \geq \frac{\pi}{4}$ (ie far from the zone) it is observed that the distribution of forces no longer compensates for the direct and image stresses, Therefore the surface is no longer free, hence the importance Of this distribution of Boussinesq forces.

\section{Edge dislocations dipoles to relax the epitaxial deformation}

Consider a film of embedded material is buried in a matrix of a semi-infinite substance with heteroepitaxial defects between the structure of the deposid thin film and the structure of the substrate.

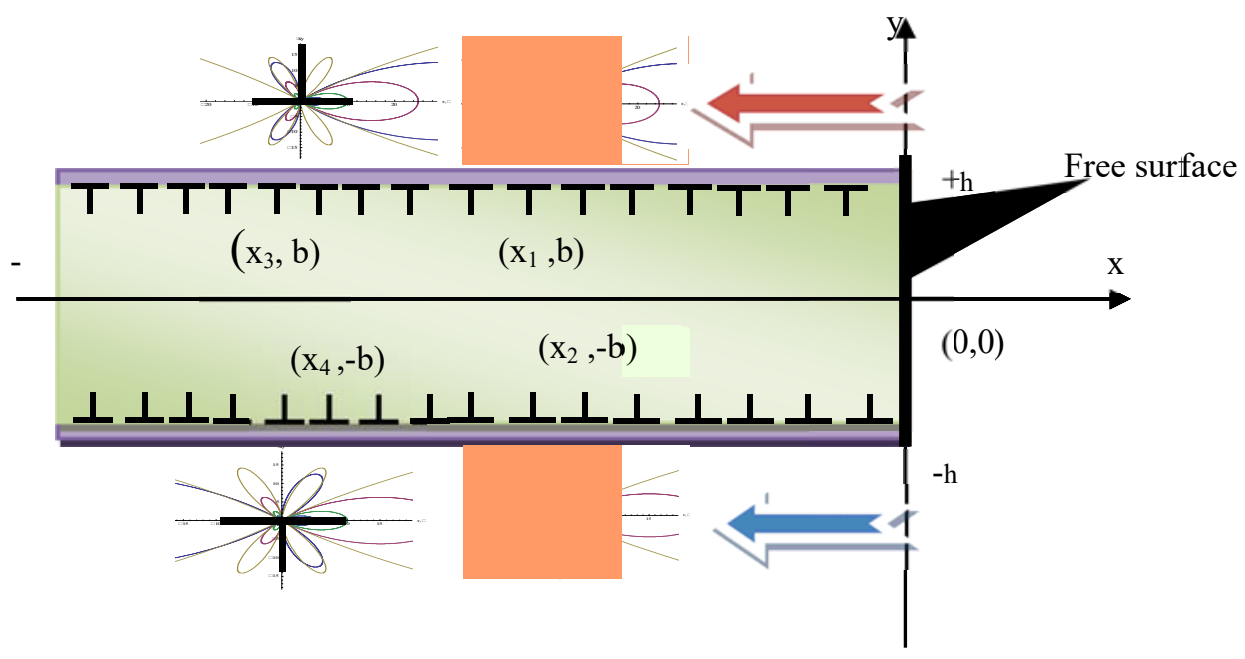

Figure 9. Nucleation of two dipoles $(1,2)$ and $(3,4)$

\subsection{Case of a single dipole (i, j)}

The first dislocation $(i,+b)$ is on the top interface $+h$ of the layer and the other $(j,-b)$ on the interface of the bottom $-h$.

Self-image stress: ${ }^{\tau_{i \leftarrow i}^{i m a g e}}=\frac{-\mu \cdot b}{4 \cdot \pi \cdot(1-v)} \cdot \frac{1}{x_{i}}$

Direct stress of the dislocation $\mathrm{j}$ :

$$
\underset{i \leftarrow j}{\tau_{i \leftarrow e c t e}^{\text {dire }}}=\frac{-\mu \cdot b}{2 \cdot \pi \cdot(1-v)} \cdot \frac{\left(x_{i}-x_{j}\right) \cdot\left(\left(x_{i}-x_{j}\right)^{2}-4 \cdot h^{2}\right)}{\left(\left(x_{i}-x_{j}\right)^{2}+4 \cdot h^{2}\right)^{2}}
$$

Impact of the image dislocation $\mathrm{j}$ on the studied dislocation I:

$\tau_{i \leftarrow j}^{\text {image }}=\frac{\mu \cdot b}{2 \cdot \pi \cdot(1-v)} \cdot \frac{\left(x_{i}+x_{j}\right) \cdot\left(\left(x_{i}+x_{j}\right)^{2}-4 \cdot h^{2}\right)}{\left(\left(x_{i}+x_{j}\right)^{2}+4 \cdot h^{2}\right)^{2}}$

Stress due to the Boussinesq forces distribution on the free surface 


$$
\tau_{i \leftarrow j}^{\text {Bsnq }}=\frac{-\mu \cdot b \cdot x_{j}}{\pi \cdot(1-v)} \cdot \frac{\left(x_{i}+x_{j}\right)^{4}-2 \cdot x_{i} \cdot\left(x_{i}+x_{j}\right)^{3}+24 \cdot x_{i} \cdot\left(x_{i}+x_{j}\right) \cdot h^{2}-16 \cdot h^{4}}{\left(\left(x_{i}+x_{j}\right)^{2}+4 \cdot h^{2}\right)^{3}}
$$

Stress due to the epitaxial deformation on the two interfaces:

$\tau_{i}^{e p i t}=\frac{\mu \cdot b^{e p i t}}{2 \cdot \pi \cdot a \cdot(1-v)} \cdot\left[\frac{4 \cdot x_{i}{ }^{2}}{x_{i}{ }^{2}+\left(y_{i}-h\right)^{2}}-\frac{4 \cdot x_{i}{ }^{2}}{x_{i}{ }^{2}+\left(y_{i}+h\right)^{2}}\right]_{[9]}$

$b^{e p i t}= \pm \delta a$ the Burgers vector relating to the deformation of epitaxy, and $\varepsilon^{e p i t}=\frac{b^{e p i t}}{a}=\frac{\delta a}{a}$

For the first dislocation of dipole: $\mathrm{i}=1\left(b, x_{1},+h\right)$

$$
\begin{aligned}
& \tau_{1}^{\text {tot }}=\tau_{1}^{\text {epit }}+\tau_{1 \leftarrow 2}^{\text {Bsnq }}+\tau_{1 \leftarrow 2}^{\text {direct }}+\tau_{1 \leftarrow 1}^{\text {image }}+\tau_{1 \leftarrow 2}^{\text {image }} \\
& \left\{\begin{array}{l}
\tau_{1}^{\text {epit }}=\frac{16 \cdot \mu}{2 \cdot \pi \cdot(1-v)} \cdot \frac{\varepsilon^{e p i t} \cdot h^{2}}{x_{1}{ }^{2}+4 \cdot h^{2}} \\
\tau_{1 \leftarrow 2}^{\text {Bsnq }}=-\frac{\mu \cdot b \cdot x_{2}}{\pi \cdot(1-v)} \cdot \frac{\left(x_{1}+x_{2}\right)^{4}-2 x_{1} \cdot\left(x_{1}+x_{2}\right)^{3}+24 \cdot x_{1} \cdot h^{2} \cdot\left(x_{1}+x_{2}\right)-16 \cdot h^{4}}{\left[\left(x_{1}+x_{2}\right)^{2}+4 \cdot h^{2}\right]^{3}} \\
\tau_{1 \leftarrow 2}^{\text {direct }}=-\frac{\mu \cdot b}{2 \cdot \pi \cdot(1-v)} \cdot \frac{\left(x_{1}-x_{2}\right)\left[\left(x_{1}-x_{2}\right)^{2}-4 \cdot h^{2}\right]}{\left[\left(x_{1}-x_{2}\right)^{2}+4 \cdot h^{2}\right]^{2}} \\
\tau_{1 \leftarrow 1}^{\text {image }}=-\frac{\mu \cdot b}{4 \cdot \pi \cdot(1-v) \cdot x_{1}} \\
\tau_{1 \leftarrow 2}^{\text {image }}=\frac{\mu \cdot b \cdot\left(x_{1}+x_{2}\right)}{2 \cdot \pi \cdot(1-v)} \cdot \frac{\left(x_{1}+x_{2}\right)^{2}-4 \cdot h^{2}}{\left(\left(x_{1}+x_{2}\right)^{2}+4 \cdot h^{2}\right)^{2}}
\end{array}\right.
\end{aligned}
$$

For the second dislocation of dipole: $\mathrm{j}=2\left(-b, x_{2},-h\right)$

$$
\begin{aligned}
& \tau_{2}^{\text {tot }}=\tau_{2}^{\text {epit }}+\tau_{2 \leftarrow 1}^{\text {Bsnq }}+\tau_{2 \leftarrow 1}^{\text {direct }}+\tau_{2 \leftarrow 2}^{\text {image }}+\tau_{2 \leftarrow 1}^{\text {image }} \\
& \text { Using the reduced coordinates: }\left\{\begin{array}{l}
B=\frac{b}{2 \cdot h} \\
k=\frac{\varepsilon^{e p i t}}{B} \\
X=\frac{x}{2 \cdot h} \\
F=f \cdot \frac{2 \cdot \pi \cdot(1-v)}{2 \cdot \mu \cdot B^{2} \cdot h}
\end{array}\right.
\end{aligned}
$$

And according to Peach Koehler's equation: $\overrightarrow{d F}=|\vec{b}|\left[\sigma_{b} \wedge \overrightarrow{d l}\right]$

$\vec{b}$ : Burgers vector and ${ }^{\overrightarrow{d l}}$ line vector dislocation line.

We have: $\left\{\begin{array}{l}f_{1}^{\text {tot }}=f_{1 \leftarrow 1}^{i m 1}+f_{1 \leftarrow 2}^{\text {im } 2}+f_{1 \leftarrow 2}^{\text {direct }}+f_{1 \leftarrow 2}^{\text {Bsnq }}+f_{1 \leftarrow}^{\text {epit }} \\ f_{2}^{\text {tot }}=f_{2 \leftarrow 1}^{i m 1}+f_{2 \leftarrow 2}^{\text {im } 2}+f_{2 \leftarrow 1}^{\text {direct }}+f_{2 \leftarrow 1}^{\text {Bsn }}+f_{2 \leftarrow}^{\text {epit }} \\ f_{i \leftarrow j}^{\alpha}=\sigma_{i \leftarrow j}^{\alpha} b \\ F_{i}^{\text {tot }}=f_{i}^{\text {tot }} \cdot \frac{2 \cdot \pi \cdot(1-v)}{2 \cdot \mu \cdot B^{2} \cdot h}\end{array}\right.$ 


$$
\begin{gathered}
F_{1}^{\text {tot }}=\frac{-1}{2 \cdot X_{1}}+\frac{\left(X_{1}+X_{2}\right) \cdot\left[\left(X_{1}+X_{2}\right)^{2}-1\right]}{\left[\left(X_{1}+X_{2}\right)^{2}+1\right]^{2}}-\left(X_{1}-X_{2}\right) \frac{\left[\left(X_{1}-X_{2}\right)^{2}-1\right]}{\left[\left(X_{1}-X_{2}\right)^{2}+1\right]^{2}}-2 \cdot X_{2} \cdot \frac{\left(X_{1}+X_{2}\right)^{4}-2 \cdot X_{1} \cdot\left(X_{1}+X_{2}\right)^{3}+6 \cdot X_{1} \cdot\left(X_{1}+X_{2}\right)-1}{\left[\left(X_{1}+X_{2}\right)^{2}+1\right]^{3}}+\frac{4 \cdot K}{1+X_{1}{ }^{2}} \\
F_{2}^{\text {tot }}=\frac{-1}{2 \cdot X_{2}}+\frac{\left(X_{1}+X_{2}\right) \cdot\left[\left(X_{1}+X_{2}\right)^{2}-1\right]}{\left[\left(X_{1}+X_{2}\right)^{2}+1\right]^{2}}-\left(X_{2}-X_{1}\right) \frac{\left[\left(X_{2}-X_{1}\right)^{2}-1\right]}{\left[\left(X_{2}-X_{1}\right)^{2}+1\right]^{2}}-2 \cdot X_{1} \cdot \frac{\left(X_{1}+X_{2}\right)^{4}-2 \cdot X_{1} \cdot\left(X_{1}+X_{2}\right)^{3}+6 \cdot X_{1} \cdot\left(X_{1}+X_{2}\right)-1}{\left[\left(X_{1}+X_{2}\right)^{2}+1\right]^{3}}+\frac{4 \cdot K}{1+X_{2}{ }^{2}}
\end{gathered}
$$

We compute the equilibrium positions of the dipole [10], initiating the calculation from $\mathrm{k}=-0.6$ and $\left(X_{01}, X_{02}\right)=(-2,-0.28)$ and we have the two curves:

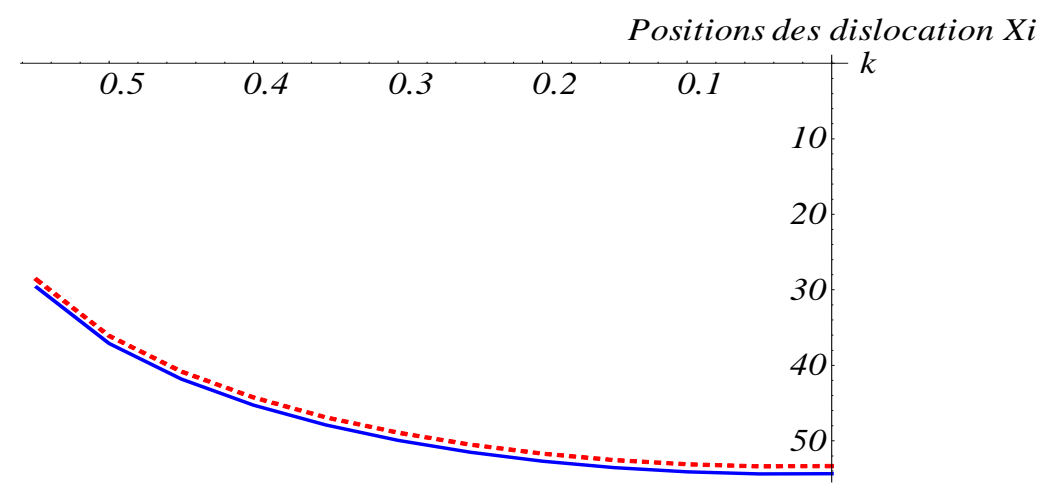

Figure 10. Equilibrium positions $X 1$ and $X 2$

\subsection{Case of two and four dipoles}

Using the same calculations, we have:

$$
\begin{aligned}
& F_{1}^{\text {tot }}=\left(X_{1}, X_{2}, X_{3}, X_{4}\right)=\frac{-1}{2 \cdot X_{1}}+\frac{4 . k}{X_{1}^{2}+1}+f_{j i}\left(X_{1}, X_{3}\right)+g_{j i}\left(X_{1}, X_{2}\right)+g_{j i}\left(X_{1}, X_{4}\right) \\
& F_{2}^{\text {tot }}=\left(X_{1}, X_{2}, X_{3}, X_{4}\right)=\frac{-1}{2 \cdot X_{2}}+\frac{4 . k}{X_{2}^{2}+1}+f_{j i}\left(X_{2}, X_{4}\right)+g_{j i}\left(X_{2}, X_{1}\right)+g_{j i}\left(X_{2}, X_{3}\right) \\
& F_{3}^{\text {tot }}=\left(X_{1}, X_{2}, X_{3}, X_{4}\right)=\frac{-1}{2 \cdot X_{3}}+\frac{4 . k}{X_{3}^{2}+1}+f_{j i}\left(X_{3}, X_{1}\right)+g_{j i}\left(X_{3}, X_{2}\right)+g_{j i}\left(X_{3}, X_{4}\right)
\end{aligned}
$$

for : $X_{i}-X_{j} \neq 0$

$$
\left\{\begin{array}{l}
g_{j i}\left(X_{i}, X_{j}\right)=\frac{\left(X_{i}+X_{j}\right) \cdot\left[\left(X_{i}+X_{j}\right)^{2}-1\right]}{\left[\left(X_{i}+X_{j}\right)^{2}+1\right]^{2}}-\frac{\left(X_{i}-X_{j}\right) \cdot\left[\left(X_{i}-X_{j}\right)^{2}-1\right]}{\left[\left(X_{i}-X_{j}\right)^{2}+1\right]^{2}}-2 \cdot X_{j} \cdot \frac{\left[\left(X_{i}+X_{j}\right)^{4}-2 \cdot X_{i} \cdot\left(X_{i}+X_{j}\right)^{3}+6 \cdot X_{i} \cdot\left(X_{i}+X_{j}\right)-1\right]}{\left[\left(X_{i}+X_{j}\right)^{2}+1\right]^{3}} \\
f_{j i}\left(X_{i}, X_{j}\right)=\frac{1}{X_{i}-X_{j}}-\frac{1}{X_{i}+X_{j}}-2 \cdot X_{j} \cdot \frac{X_{j}-X_{i}}{\left(X_{i}+X_{j}\right)^{3}}
\end{array}\right.
$$

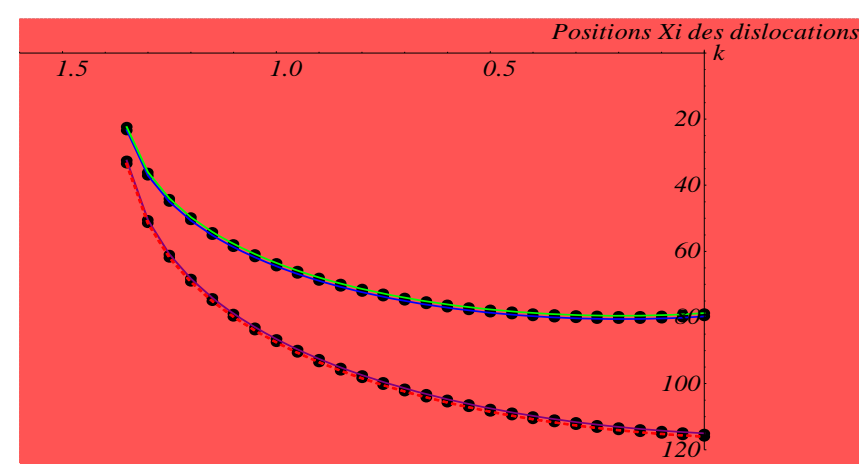

Figure 11. The four curves of the four equilibrium positions $X 1, X 2, X 3$ and $X 4$ 


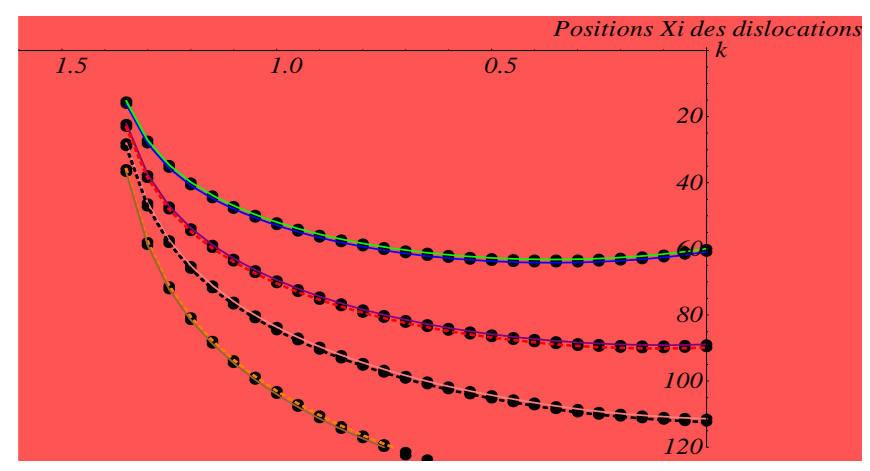

Figure 12. Case of 4 dipoles

\section{Conclusion}

In conclusion, and after calculating the shear stresses due to residual defects caused by the mesh parameter defects at the lateral surface in the interface intersection with the thin film - substrate, we canceled their effects by adding to the image dislocations a Boussinesq distribution ponctual and unit forces after which a collection of edge dislocations dipoles is issued from the surface. We have shown their primordial importance to relax the instability of the material through the calculation of their stable equilibrium positions, which will in no case leave the matrix of the material. We have worked on a lateral crossing surface normal to the principal axis Ox of the material; we leave as a first perspective the case where the surface will have any inclination, as well as the case of a finite material and surroundings its borders.

\section{Références}

[1] Stress relaxation and misfit dislocation nucleation in the growth of misfitting films, Liang Dong, Jurgen Schnitker, Richard W. Smith,a) and David J. Srolovitzb) Department of Materials Science and Engineering, University of Michigan, Ann Arbor, Michigan 48109-2136. 1998 American Institute of Physics. [S0021-8979(98)05401-2]

[2] Matthews, "Defects associated with the accommodation of misfit between crystals, J. Vac. Sci. Technol. 12; 126(1975).

[3] Anandh Subramaniama.Critical thickness of equilibrium epitaxial thin films using finite element method. Journal of Applied Physics.Volume 95, Number 12

[4] Head.A.K. (1953). Proc.Phy. Soc. Lond. B 66, 793.

[5] K.L.JOHNSON Emeritus Professor of Engineering, University of Cambridge. Cambridge University Press 1985

[6] S.P. Timoshenko and J.N. Goodier, "Theory of Elasticity,” McGraw-Hill, Third Ed., New York, (1970)

[7] John Price Hirth, Jens Loth. Department of metallurgical Engineering The Ohio state University, Institute of physics, Oslo University. Second Edition. 1982

[8] Adhesion increase by interface mixing. J. Colin , N. Junqua \& J. Grilhé. Philosophical Magazine A. 75:2, 369-377.20 Aug 2006.

[9] XLI. The equilibrium of linear arrays of dislocations. J.D. Eshelby a , F.C. Frank \& F.R.N. Nabarro (1951). Philosophical Magazine Series 7, 42:327, 351-364

[10] Surface Instabilities and Dislocation Formation at Free Surfaces of Stressed Solids. J. GRILHE Laboratoire de Métallurgie Physique, U.A. 131 du CNRS, Université 40, av. du Recteur Pineau, 86022 Poitiers, France. Europhys. Lett., 23 (21, pp. 141-146 (1993). IOP science

[11] Plastic Folding of Buckling Structures. Jérôme Colin,* Christophe Coupeau, and Jean Grilhé Laboratoire de Métallurgie Physique, UMR 6630 du CNRS, Université de Poitiers, France. PHYSICAL REVIEW LETTERS. PRL 99, 046101 (2007)

[12] William D.Nix. Mechanical Properties of Thin Films. Stanford University, 2005. 
[13] Zhu et al. 2008, Temperature and Strain-Rate Dependence of Surface Dislocation Nucleation, (C 2008 The American Physical Society,PRL 100, 025502 (2008).

[14] Anandh Subramaniama.Critical thickness of equilibrium epitaxial thin films using finite element method. Journal of Applied Physics.Volume 95, Number 12

[15] D. HULL AND D. J. BACON: INTRODUCTION TO DISLOCATIONS, FOURTH ED, (BUTTERWORTH HEINEMANN).

[16] Ohno S. D. Hull, J. Bacon. Introduction to Dislocations, Fourth Edition. University of Liverpool, UK. [4] R.Hill : The mathematical theory of plasticity, Oxford 1998.

[17] I.S.Sokolnikoff. Mathematical Theory of Elasticity, University of California, 1946.

[18] A.M. Kossevich, the Crystal Lattice: Phonons, Solitons, Dislocations, 1999.

[19] Chi-Chin Wu, Eric A Stach and Robert Hull. Nanoscale mechanisms of misfit dislocation propagation in undulated Sil- xGex/Si (100) epitaxial thin films.IOP publishing journal. Nanotechnology 18 (2007) 165705.

[20] Tomotsugu Shimokawa and Soya Kitada, Dislocation Multiplication from the Frank Read Source in Atomic Models, Materials Transactions, Vol.55 (2014).

[21] F. C. Frank andW. T. Read, Jr: Phys. Rev. 79(1950). [11] H. H. Wang, S. Y. Byrapa, F. Wu, B. Raghothamachar, M. Dudley, E. K. Sanchez, D. M. Hansen, R. Drachev, S. G. Mueller, M. J. Loboda, "Basal Plane Dislocation Multiplication via the Hopping Frank-Read Source Mechanism and Observations of Prismatic Glide in 4H-SiC", Materials Science Forum, Vols. 717 - 720, May:2012. 